\title{
EFEITOS DO TREINAMENTO AERÓBICO E DO FORTALECIMENTO EM PACIENTES COM INSUFICIÊNCIA CARDIIACA
}

\author{
EFFECTS OF AEROBIC TRAINING AND STRENGTHENING IN PATIENTS WITH HEART FAILURE \\ EFECTOS DEL ENTRENAMIENTO AERÓBICO Y DEL FORTALECIMIENTO EN PACIENTES CON \\ INSUFICIENCIA CARDÍACA
}

Leonardo Calegari' (Fisioterapeuta)

Bibiana Ferrari Barroso ${ }^{1}$

(Acadêmica do Curso de Fisioterapia)

Juliete Bratz ${ }^{1}$

(Acadêmica do Curso de Fisioterapia)

Sara Romano' (Fisioterapeuta)

Gabriela Forcelini de Figueiredo

(Fisioterapeuta)

Marina Ceccon' (Fisioterapeuta)

Gilnei Lopes Pimentel'

(Fisioterapeuta)

José Basileu Caon Reolão²

(Médico Cardiologista)

1. Universidade de Passo Fundo, Faculdade de Educação Física e Fisioterapia, Passo Fundo, RS, Brasil.

2. Universidade de Passo Fundo,

Faculdade de Medicina, Passo

Fundo, RS, Brasil.

\section{Correspondência:}

Faculdade de Educação Física e Fisioterapia - Universidade de Passo Fundo Campus I - BR 285, Bairro São José, Passo Fundo, RS, Brasil. 99052-900. leocalegari@upf.br

\section{RESUMO}

Introdução: A intolerância ao exercício e a dispneia são os principais sintomas da insuficiência cardíaca (IC). Objetivo: Avaliar os efeitos de um programa de exercícios aeróbicos e de fortalecimento sobre a aptidão cardiorrespiratória, o pico de torque dos flexores e extensores de joelho e a qualidade de vida de pacientes com IC. Métodos: Estudo prospectivo, com avaliação pré e pós-reabilitação cardiovascular (RCV) de sete pacientes, com idade de $61 \pm 6$ anos, classe funcional II e III e fração de ejeção do ventrículo esquerdo 45,4 $\pm 2,3 \%$. O programa de RCV consistiu em 24 sessões de 60 minutos com treinamento aeróbico na intensidade do limiar de anaerobiose (LA) e fortalecimento dos membros inferiores usando caneleiras de 3 a $5 \mathrm{~kg}$. No início e após RCV os pacientes realizaram prova de esforço, dinamometria isocinética do joelho dominante e responderam o questionário WHOQOL-bref. Resultados: Após RCV, o tempo de exercício para atingir o LA foi atrasado $(p=0,04)$ e houve aumento significativo no consumo de oxigênio $\left(\mathrm{VO}_{2}\right)(p<0,01)$, da frequência cardíaca $(F C)(p=0,04)$, pulso de oxigênio $\left(V_{2} / F C\right)(p=0,02)$ e ventilação $(V E)(p=0,01)$ na intensidade do $L A$. Houve aumento do pico de torque dos músculos extensores de joelho $(p=0,02)$ e melhora significativa do domínio psicológico $(p=0,04)$ do questionário de qualidade de vida. Conclusão: O programa de RCV foi seguro e resultou em melhora do desempenho de exercícios submáximos, da força dos músculos extensores de joelho e da qualidade de vida de pacientes com IC.

Descritores: insuficiência cardíaca; reabilitação; limiar anaeróbio; dinamômetro de força muscular; qualidade de vida.

\section{ABSTRACT}

Introduction: Exercise intolerance and dyspnea are the main symptoms of heart failure (HF). Objective: To evaluate the effects of a program of aerobic exercises and strengthening on cardiorespiratory fitness, maximum torque of knee flexors and extensors, and quality of life of patients with HF. Methods: Prospective, pre- and post-cardiovascular rehabilitation (CVR) study of seven patients, aged $61 \pm 6$ years, functional class II and III and left ventricular ejection fraction $45.4 \pm 2.3 \%$. The CVR program consisted of 24 sessions of 60 minutes with aerobic training in intensity of the anaerobic threshold (AT) and strengthening of the lower limbs using ankle weights of 3 to $5 \mathrm{~kg}$. At the beginning and after CVR, the patients performed stress test, isokinetic dynamometry of the dominant knee and completed the WHOQOL-bref questionnaire. Results: After CVR, exercise time to reach AT was delayed $(p=0.04)$ and there was a significant increase in oxygen consumption $\left(\mathrm{VO}_{2}\right)(p<0.01)$, heart rate $(\mathrm{HR})(p=0.04)$, pulse of oxygen $\left(\mathrm{VO}_{2} / \mathrm{HR}\right)(p=0.02)$ and ventilation (VE) $(p=0.01)$ in the intensity of AT. There was an increase in maximum torque of knee extensor muscles $(p=0.02)$ and significant improvement in the psychological domain ( $p=0.04$ ) of the quality of life questionnaire. Conclusion: The CVR program was safe and resulted in improved performance of submaximal exercises, knee extensor muscles strength and quality of life of patients with $\mathrm{HF}$.

Keywords: heart failure; rehabilitation; anaerobic threshold; muscle strength dynamometer; quality of life.

\section{RESUMEN}

Introducción: La intolerancia al ejercicio y la disnea son los síntomas principales de la insuficiencia cardiaca (IC). Objetivo: Evaluar los efectos de un programa de ejercicio aeróbico y fortalecimiento en la capacidad cardiorrespiratoria, el par máximo de los flexores y extensores de rodilla y la calidad de vida de los pacientes con IC. Métodos: Estudio prospectivo, con evaluación pre y post-rehabilitación cardiovascular (RCV) de siete pacientes, edad $61 \pm 6$ años, clase funcional IIy III y fracción de eyección del ventrículo izquierdo de 45,4 2,3\%. El programa de RCV consistió en 24 sesiones de 60 minutos con la intensidad del entrenamiento aeróbico en el umbral de anaerobiosis (UA) y el fortalecimiento de las extremidades inferiores utilizando pesos de tobillo de 3 a $5 \mathrm{~kg}$. Al principio y después de la RCV los pacientes se sometieron a la prueba de esfuerzo, dinamometría isocinética de la rodilla dominante y respondieron el cuestionario WHOQOL-bref. Resultados: Después de la RCV, el tiempo de ejercicio para alcanzar UA se retrasó $(p=0,04)$ y hubo un aumento significativo en el consumo de oxígeno $\left(V_{2}\right)(p<0,01)$, frecuencia cardiaca $(F C)(p=0,04)$, pulso de oxígeno $\left(\mathrm{VO}_{2} / F C\right)(p=0,02)$ y la ventilación (VE) $(p=0,01)$ en la intensidad del UA. Hubo un aumento del par máximo de los músculos extensores de la rodilla $(p=0,02)$ y una mejora significativa en el dominio psicológico $(p=0,04)$ del cuestionario 
de calidad de vida. Conclusión: El programa de RCV fue seguro y mejoró el rendimiento de los ejercicios submáximos, la fuerza de los músculos extensores de la rodilla y la calidad de vida de los pacientes con IC.

Descriptores: insuficiencia cardiaca; rehabilitación; umbral anaerobio; dinamómetro de fuerza muscular; calidad devida.

\section{INTRODUÇÃO}

A insuficiência cardíaca (IC) tem sido considerada uma epidemia e projeções mostram que a prevalência de IC aumentará 46\% de 2012 - 2030, resultando em mais de 8 milhões de pessoas com IC nos Estados Unidos'. Em 2012, segundo os dados do DATA-SUS, houve 1.137.572 internações por doenças cardiovasculares e aproximadamente $21 \%$ foram relacionadas à $I C^{2}$. Esse fato se deve ao crescente envelhecimento populacional e alta expectativa de vida, o que em conjunto com a otimização do tratamento clínico, contribuiu para o aumento e a prevalência da IC ${ }^{3}$. A IC é caracterizada pela incapacidade do coração em oferecer uma perfusão adequada para atender as necessidades metabólicas do corpo, representando a via final de várias cardiopatias ${ }^{2}$. Sua principal característica refere-se a intolerância ao exercício, levando a sintomas de fadiga, dispneia, baixos escores de qualidade de vida e a uma redução no consumo de oxigênio de pico $\left(\mathrm{VO}_{2 \text { pico }}\right)$, se comparado com indivíduos saudáveis ${ }^{4}$.

Embora a IC apresente uma disfunção cardíaca, tem sido sugerido que a intolerância ao exercício, refere-se em grande parte a alterações na musculatura esquelética. Tais alterações incluem baixo fluxo sanguíneo, capacidade reduzida do músculo em utilizar oxigênio, aumento dos níveis de citocinas pró-inflamatórias e aumento do estresse oxidativo ${ }^{5}$. O descondicionamento físico contribui para a sensação de fadiga usualmente relatada pelos pacientes com IC ${ }^{6}$. Portanto, a intolerância ao exercício se deve tanto as alterações cardiovasculares quanto as alterações musculoesqueléticas ${ }^{6}$.

Programas de reabilitação cardiovascular (RCV) baseiam-se em uma abordagem individualizada dos pacientes, realizada por uma equipe capaz de prescrever exercício físico e de fortalecimento muscular, orientar sobre hábitos alimentares saudáveis e estimular a prática de atividades físicas ${ }^{7,8}$. Os programas de RCV que seguem as diretrizes são relativamente seguros apresentando 1 evento para 112.000 pacientes/ hora de exercícios ${ }^{8}$. Apesar da RCV ser considerada segura e com bons indicadores de custo-efetividade, o encaminhamento por parte dos cardiologistas é muito baixo ${ }^{8-10}$. A inserção de pacientes aos programas de exercícios pode acrescentar ao tratamento farmacológico, melhorar a qualidade de vida e reduzir a intolerância ao esforço $0^{9,10}$.

Os efeitos do exercício físico regular e suas adaptações fisiológicas possuem grande importância na manutenção da saúde dessa população. O treinamento aeróbico associado ao fortalecimento muscular promoveu aumento da distância percorrida no teste de caminhada de seis minutos e melhora na qualidade de vida de pacientes submetidos à cirurgia de revascularização do miocárdio ${ }^{7}$. Nos últimos anos, o fortalecimento muscular passou a fazer parte dos programas de RCV, contribuindo para a melhora da aptidão cardiorrespiratória $^{8,9}$. Entretanto, poucos estudos utilizam o teste cardiopulmonar e a dinamometria isocinética na avaliação dos pacientes com IC após programas de RCV.

Neste contexto, este estudo teve como objetivo avaliar os efeitos de um programa de exercícios aeróbicos e de fortalecimento sobre a aptidão cardiorrespiratória, o pico de torque dos flexores e extensores de joelho e a qualidade de vida de pacientes com IC.

\section{MATERIAIS E MÉTODOS}

O projeto foi aprovado pelo Comitê de Ética em Pesquisa sob o parecer no 004/2011, todos os pacientes aceitaram participar da pesquisa e assinaram um Termo de Consentimento Livre e Esclarecido. Estudo experimental prospectivo, com avaliação pré e pós RCV de oito pacientes, sendo cinco homens e três mulheres, classificados como classe funcional II e III, segundo a New York Heart Association (NYHA) ${ }^{11}$. O estudo foi conduzido na Faculdade de Educação Física e Fisioterapia da Universidade de Passo Fundo e os pacientes foram encaminhados pelos cardiologistas. Foram incluídos no estudo pacientes com diagnostico de IC há mais de um ano, clinicamente estáveis nos últimos três meses, com fração de ejeção do ventrículo esquerdo < 50\%. Os critérios de exclusão foram: mudança de medicamentos, instabilidade hemodinâmica, disfunção ortopédica ou neurológica, doença pulmonar crônica ou insuficiência renal crônica. Uma paciente foi excluída da amostra por necessitar de internação hospitalar.

A avaliação inicial foi realizada por um médico cardiologista que estratificou o risco que o exercício representava para cada paciente. Foi preenchida uma ficha de avaliação contendo medicamentos em uso, peso e altura, interpretação de exames complementares para caracterização da amostra e finalizado com um eletrocardiograma de repouso.

O teste cardiopulmonar (TECP) foi em esteira rolante (Master ATL, Inbramed) utilizando protocolo de rampa, com incrementos de carga linear e progressivo até a exaustão física e/ou o surgimento de sinais e sintomas limitantes ${ }^{12,13}$. Durante o teste os indivíduos foram monitorizados continuamente em 12 derivações eletrocardiográficas e analisados os gases expirados em circuito aberto (Software Ergo PC Elite VO 2000). A verificação da pressão arterial (PA) e a quantificação subjetiva da percepção de esforço de Borg (6 a 20 pontos) foram registradas em intervalos de três minutos. A determinação do limiar de anaerobiose $(L A)$ foi realizada pela técnica ventilatória ${ }^{13}$. Os valores apresentados para o LA e pico de esforço foram calculados em médias de 40 segundos. A frequência cardíaca mensurada na intensidade correspondente ao limiar de anaerobiose ( $\left.F C_{L A}\right)$ foi utilizada na prescrição da intensidade do exercício aeróbico ${ }^{10}$. O teste foi finalizado assim que o sujeito solicitou, por fadiga ou quaisquer dos critérios indicados para interrupção do teste, definidos pelas diretrizes da Sociedade Brasileira de Cardiologia14.

O pico de torque foi avaliado com dinamômetro isocinético da marca Biodex ${ }^{\mathrm{TM}}$ Multi Joint System 3 Pro. Antes da avaliação isocinética, os indivíduos realizaram exercícios de aquecimento e alongamento dos membros inferiores. A PA foi mensurada pré e pós-avaliação. $\mathrm{O}$ eixo de rotação do dinamômetro foi alinhado com o eixo da articulação do joelho. Os participantes foram solicitados a realizar 5 contrações consecutivas máximas para flexão e extensão do joelho dominante à $60 \%$ segundo ${ }^{15}$.

Utilizou-se o instrumento de avaliação da qualidade de vida abreviado da Organização Mundial da Saúde (WHOQOL-bref) traduzido e validado para a versão em português. O questionário WHOQOL-bref é composto por 26 questões divididas em 4 domínios: físico, psicológico, relações sociais e meio ambiente. Seus domínios são pontuados de 0 a 100 pontos, quanto maior o escore melhor é a qualidade de vida ${ }^{16}$.

O protocolo de RCV foi composto por três sessões semanais com duração de 60 minutos cada, por um período de oito semanas 
(24 sessões). As sessões foram divididas em condicionamento aeróbico e força muscular periférica. O condicionamento aeróbico consistiu em caminhadas em esteira ou em pista de atletismo por um período de 30 minutos. A intensidade do exercício foi prescrita através da FC atingida no LA, obtido através do TECP para cada individuo e monitorada através de um cardiofrequencímetro da marca Polar ${ }^{\oplus 10}$. O protocolo de fortalecimento muscular para os membros inferiores consistiu de exercícios de flexão/extensão e movimentos em diagonais de joelho, quadril e tornozelo com caneleiras durante 30 minutos. Nas duas primeiras semanas foram utilizados 2-3 kg e a partir da 3a semana $4-5 \mathrm{~kg}$ com 3-5 séries de 8-15 repetições que foram aumentando no decorrer das semanas. Antes e após cada sessão foi realizado alongamento ativo de membros superiores e inferiores. Durante a execução dos exercícios de fortalecimento a percepção do esforço foi monitorada entre 11 a 15 na escala de Borg ${ }^{17}$.

\section{Análise estatística}

As variáveis quantitativas contínuas foram expressas em medidas e desvio padrão e as variáveis categóricas expressas em valor absoluto e porcentagem. O teste de Kolmogorov-Smirnov foi utilizado para analisar a distribuição dos dados. As comparações entre os dois momentos (prée pós-reabilitação) foram calculados pelo teste t de Student pareado ou o teste de Wilcoxon para distribuiç̧ões assimétricas. O nível de significância utilizado foi de $p<0,05$.

\section{RESULTADOS}

As características gerais dos pacientes e a terapia farmacológica utilizada estão descritas na Tabela 1. Nossa amostra foi composta por sete indivíduos, cinco do sexo masculino e dois do sexo feminino. A idade média foi de 61,6 6 6,1 anos e fração de ejeção do ventrículo esquerdo de $45,4 \pm 2,3 \%$. Todos os participantes completaram as 24 sessões propostas e não houve intercorrências.

Após $R C V$, os resultados do TECP avaliados na intensidade do LA (Tabela 2) mostram aumento significativo nos seguintes parâmetros: tempo para atingir o LA (291 \pm 81s para $346 \pm 69 \mathrm{~s} p=0,04)$, FC (103 \pm $15 \mathrm{bpm}$ para $111 \pm 14 \mathrm{bpm} ; \mathrm{p}=0,04), \mathrm{VO}_{2}$ absoluto $\left(1,28 \pm 0,2 \mathrm{~L} \cdot \mathrm{min}^{-1}\right.$ para 1,47 $\left.\pm 0,2 \mathrm{~L} \cdot \mathrm{min}^{-1} ; \mathrm{p}=0,01\right), \mathrm{VO}_{2}$ relativo $\left(15,1 \pm 3,5 \mathrm{ml} \cdot \mathrm{kg}^{-1} \cdot \mathrm{min}^{-1}\right.$ para $\left.17,2 \pm 3 \mathrm{ml} \cdot \mathrm{kg}^{-1} \cdot \mathrm{min}^{-1} ; \mathrm{p}=0,01\right), \mathrm{VO}_{2} / \mathrm{FC}$ pulso de oxigênio $(12,5 \pm$ 2,3 ml.bpm $^{-1}$ para $13,7 \pm 2,4$ ml.bpm $\left.^{-1} ; \mathrm{p}=0,02\right)$ e VE $\left(25,1 \pm 5,8{\mathrm{~L} . \mathrm{min}^{-1}}^{-1}\right.$ para 32,8 $\pm 5,7 \mathrm{~L}$. $\left.\mathrm{min}^{-1} ; \mathrm{p}=0,01\right)$. Após $\mathrm{RCV}$, os resultados do TECP avaliados na intensidade do esforço máximo (Tabela 3) mostram aumento significativo nos seguintes parâmetros: FC (118 \pm 16 bpm para $127 \pm 14$ bpm; $p=0,04)$ e VE $\left(39,9 \pm 10,6\right.$ L. min $^{-1}$ para 47,9 $\pm 9,6$ L. $\left.\mathrm{min}^{-1} ; \mathrm{p}=0,01\right)$.

A avaliação isocinética revelou aumento significativo no pico de torque dos músculos extensores de joelho (132,1 \46,9 NM para 152,9

Tabela 1. Características demográficas e clínicas dos pacientes avaliados.

\begin{tabular}{c|c}
\hline Características & Pacientes ( $\mathbf{n = 7})$ \\
\hline Idade (anos) & $61,6 \pm 6,1$ \\
\hline Homens / Mulheres & $5 / 2$ \\
\hline IMC (Kg/m $\left.{ }^{2}\right)$ & $29,1 \pm 4,8$ \\
\hline FEVE (\%) & $45,4 \pm 2,3$ \\
\hline Medicamentos & $7(100)$ \\
\hline Betabloqueador (\%) & $6(85,7)$ \\
\hline Inibidor da ECA (\%) & $5(71,4)$ \\
\hline Anticoagulante (\%) & $3(42,8)$ \\
\hline Estatinas (\%) & $2(28,5)$ \\
\hline Diurético (\%) &
\end{tabular}

$\overline{\text { Dados apresentados em média } \pm \text { desvio padrão ou valor absoluto (\%). IMC: Índice de massa corporal; FEVE: fração }}$ de ejeção do ventrículo esquerdo; ECA: enzima conversora de angiotensina. $\pm 41,5 \mathrm{NM} ; \mathrm{p}=0,02)$ após RCV, como monstra a Figura 1. Entretanto, não houve diferença significativa nos valores do pico de torque dos músculos flexores do joelho.

Em relação a qualidade de vida, observou-se ganho significativo no domínio psicológico $(59,5 \pm 10,4$ para $69,6 \pm 8,9 ; p=0,04)$. Entretanto, não foram observadas diferenças nos demais domínios como demonstrado na Tabela 4.

\section{DISCUSSÃO}

Nossos principais achados foram relacionados com a melhora no desempenho de exercícios submáximos, como observado pelo aumento do tempo de exercício até o LA após 24 sessões de um programa de RCV que associou exercícios aeróbicos e de fortalecimento muscular em pacientes com IC. Além disso, houve melhora do pico de torque dos músculos extensores de joelho e no domínio psicológico do questionário de qualidade de vida.

Tabela 2. Valores (Média \pm DP) obtidos no TECP na intensidade correspondente ao limiar de anaerobiose (LA), pré e pós-RCV.

\begin{tabular}{c|c|c|c|c}
\hline Variáveis & Pré & Pós & Delta & Valor $\mathbf{p}$ \\
\hline Tempo $(\mathrm{s})$ & $291 \pm 81$ & $346 \pm 69$ & $55 \pm 41$ & $0,04^{*}$ \\
\hline Velocidade $(\mathrm{Km} / \mathrm{h})$ & $3,97 \pm 1$ & $4,37 \pm 0,7$ & $0,4 \pm 0,5$ & 0,08 \\
\hline Inclinação (\%) & $7,86 \pm 2,5$ & $9,1 \pm 2,7$ & $1,2 \pm 1,4$ & 0,07 \\
\hline $\mathrm{FC}(\mathrm{bpm})$ & $103 \pm 15$ & $108 \pm 14$ & $5 \pm 5$ & $0,04^{*}$ \\
\hline $\mathrm{VO}_{2}\left(\mathrm{~L} \cdot \mathrm{min}^{-1}\right)$ & $1,28 \pm 0,2$ & $1,47 \pm 0,2$ & $0,18 \pm 0,1$ & $0,01^{*}$ \\
\hline $\mathrm{VO}_{2}\left(\mathrm{ml}^{-1} \mathrm{~kg}^{-1} \cdot \mathrm{min}^{-1}\right)$ & $15,1 \pm 3,5$ & $17,2 \pm 3$ & $2,17 \pm 1$ & $0,01^{*}$ \\
\hline $\mathrm{VO}_{2} / \mathrm{FC}\left(\mathrm{ml}^{\mathrm{bmpm}}{ }^{-1}\right)$ & $12,5 \pm 2,3$ & $13,7 \pm 2,4$ & $1,2 \pm 1$ & $0,02^{*}$ \\
\hline $\mathrm{VE}\left(\mathrm{L} \cdot \mathrm{min}^{-1}\right)$ & $25,1 \pm 5,8$ & $32,8 \pm 5,7$ & $7,7 \pm 5$ & $0,01^{*}$ \\
\hline
\end{tabular}

TECP: teste de esforço cardiopulmonar; $\mathrm{RCV}$ : reabilitação cardiovascular; $\mathrm{FC}$ : frequência cardíaca; $\mathrm{VO}_{2}$ : consumo de oxigênio; $\mathrm{VO}_{2} / \mathrm{FC}$ : pulso de oxigênio; $\mathrm{VE}$ : volume minuto expirado. Delta: diferença entre pré e pós- $\mathrm{RCV}$, ${ }^{*} \mathrm{p}<0,05$ comparado aos valores pré-RCV.

Tabela 3. Valores (Média \pm DP) obtidos no TECP no esforço máximo, pré e pós-RCV.

\begin{tabular}{|c|c|c|c|c|}
\hline Variáveis & Pré & Pós & Delta & Valor $p$ \\
\hline Tempo (s) & $452 \pm 156$ & $495 \pm 112$ & $43 \pm 89$ & 0,25 \\
\hline Velocidade $(\mathrm{Km} / \mathrm{h})$ & $4,9 \pm 1,3$ & $5,3 \pm 1$ & $0,4 \pm 0,5$ & 0,06 \\
\hline Inclinação (\%) & $10,7 \pm 3,9$ & $12,2 \pm 3,8$ & $1,5 \pm 1,8$ & 0,07 \\
\hline $\mathrm{FC}(\mathrm{bpm})$ & $118 \pm 16$ & $127 \pm 14$ & $9 \pm 10$ & $0,04^{*}$ \\
\hline $\mathrm{VO}_{2}\left(\mathrm{~L} \cdot \mathrm{min}^{-1}\right)$ & $1,62 \pm 0,3$ & $1,77 \pm 0,3$ & $0,15 \pm 0,1$ & 0,06 \\
\hline $\mathrm{VO}_{2}\left(\mathrm{ml} \cdot \mathrm{kg}^{-1} \cdot \mathrm{min}^{-1}\right)$ & $19 \pm 4,9$ & $20,8 \pm 3,9$ & $1,8 \pm 2$ & 0,06 \\
\hline $\mathrm{VO}_{2} / \mathrm{FC}\left(\mathrm{ml}^{\prime} \mathrm{bpm} \mathrm{m}^{-1}\right)$ & $13,7 \pm 2,9$ & $14 \pm 2,4$ & $0,3 \pm 1,2$ & 0,63 \\
\hline VE $\left(\right.$ L.min $\left.{ }^{-1}\right)$ & $39,9 \pm 10,6$ & $47,9 \pm 9,6$ & $7,9 \pm 5,2$ & $0,01^{*}$ \\
\hline $\mathrm{VE} / \mathrm{NCO}_{2}$ & $26,2 \pm 1,1$ & $26 \pm 1,7$ & $-0,2 \pm 1,6$ & 0,77 \\
\hline$R$ & $1,05 \pm 0,07$ & $1,1 \pm 0,1$ & $0,05 \pm 1$ & 0,3 \\
\hline
\end{tabular}

TECP: teste de esforço cardiopulmonar; $\mathrm{RCV}$ : reabilitação cardiovascular; $\mathrm{FC}$ : frequência cardíaca; $\mathrm{VO}_{2}$ : consumo de oxigênio; $\mathrm{VO}_{2} / \mathrm{FC}$ : pulso de oxigênio; $\mathrm{VE}$ : volume minuto expirado. $\mathrm{VE} / \mathrm{NCO}_{2}$ : equivalente ventilatório de dióxido de carbono; $R$ : razăo de troca respiratória; Delta: diferença entre pré e pós $R C V$, ${ }^{*} p<0,05$ comparado aos valores pré-RCV.

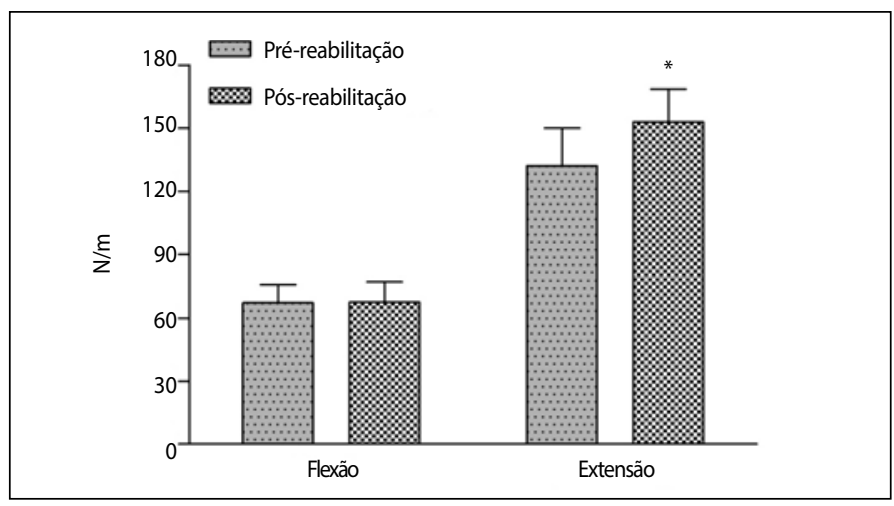

Figura 1. Pico de torque (Nm) dos flexores e extensores de joelho na velocidade de $60 \%$, pré e pós-reabilitação cardiovascular (RCV) em pacientes com insuficiência cardíaca. ${ }^{*} \mathrm{p}<0,05$ comparado aos valores pré-RCV. 
Tabela 4. Valores (Média \pm DP) nos domínios avaliados pelo questionário WHOQOL-bref, pré e pós-RCV.

\begin{tabular}{c|c|c|c|c}
\hline Domínios & Pré & Pós & Delta & Valor $\boldsymbol{p}$ \\
\hline Físico & $55,1 \pm 14,4$ & $61,7 \pm 16,1$ & $6,6 \pm 9,3$ & 0,11 \\
\hline Psicológico & $59,5 \pm 10,4$ & $69,6 \pm 8,9$ & $10,1 \pm 10$ & $0,04^{*}$ \\
\hline Social & $70,2 \pm 14,3$ & $77,4 \pm 17,2$ & $7,1 \pm 7,5$ & 0.06 \\
\hline Meio Ambiente & $64,7 \pm 9,8$ & $64,8 \pm 9,4$ & $0,1 \pm 6,6$ & 0,97 \\
\hline${ }^{*}$ p $<0,05$ comparado aos valores pré-RCV.
\end{tabular}

$\mathrm{OVO}_{2 \text { pico }}$ é o melhor marcador da capacidade funcional em pacientes com IC, além de ser um preditor independente de mortalidade ${ }^{8}$. Em nossa amostra, o VO $\mathrm{V}_{\text {2pico }}$ pós intervenção foi em média de $20,5 \mathrm{ml} \cdot \mathrm{kg}^{-1} \cdot \mathrm{min}^{-1}$. Pereira et al. ${ }^{18}$, avaliaram pacientes com classe funcional II da NYHA que apresentaram $\mathrm{VO}_{2 \text { pico }}$ de $27 \mathrm{ml} . \mathrm{kg}^{-1} \cdot \mathrm{min}^{-1}$ e pacientes com classe funcional III VO $\mathrm{O}_{2 \text { ico }}$ de $18 \mathrm{ml} . \mathrm{kg}^{-1} \cdot \mathrm{min}^{-1}$ e não observaram correlação entre $\mathrm{VO}_{2 \text { pico }}$ e a fração de ejeção. Weber et al. ${ }^{19}$ relataram que valores de $\mathrm{VO}_{2 \text { pico }}$ acima de $20 \mathrm{ml} \cdot \mathrm{kg}^{-1} \cdot \mathrm{min}^{-1}$ caracterizam melhor condição funcional em pacientes com IC. Em nosso estudo, o programa de exercícios proposto (intensidade/volume) não promoveu aumento significativo no $\mathrm{VO}_{2 \text { pico, }}$ embora observamos melhora em níveis submáximos, indicando que o treinamento físico na intensidade do LA promove adaptações metabólicas. A avaliação não invasiva do LA determinado pela técnica ventilatória representa o aumento de lactato e consequente liberação de $\mathrm{CO}_{2}$, sugerindo que os resultados do presente estudo possam ter promovido adaptações na síntese/liberação de lactato ${ }^{20}$. O LA representa o ponto de transição entre a predominância do metabolismo aeróbico, para a predominância do metabolismo anaeróbico durante o esforço físico mais intenso ${ }^{13}$. Ainda, observou-se que no período pré intervenção, os indivíduos atingiram o LA a 79\% do $\mathrm{VO}_{2 \text { pico }}$ e no período pós intervenção a média foi de 83,9\% do $\mathrm{VO}_{2 \text { pico, }}$ demonstrando que a intervenção proposta, acarretou maior tolerância aos esforços submáximos. Além disso, a identificação do LA permite uma prescrição individualizada da intensidade dos exercícios em pacientes com doenças cardiorrespiratórias ${ }^{21}$.

Estudos relatam que o treinamento físico promove redução da atividade nervosa simpática, melhora do balanço simpato/vagal e reduz a incidência de $\operatorname{arritmias}^{10}$. Em razão da diminuição da atividade simpática muscular e, em consequência da resistência vascular, ocorre um aumento no fluxo sanguíneo, resultando em uma melhor capacidade funcional ${ }^{13}$. Em um exercício dinâmico, onde as cargas aumentam progressivamente, a FC aumenta de forma linear mediante a retirada vagal e aumento do sistema simpático ${ }^{21}$. O aumento é proporcional a carga de trabalho imposta, sendo influenciado por outros fatores como idade, beta-bloqueadores e nível de condicionamento físico. Em nosso estudo, a resposta cronotrópica (RC) mensurada no TECP em vigência de beta-bloqueadores, foi em média de 37 bpm na avaliação inicial e 44 bpm na avaliação final, valores muito próximos aos relatado por Colucci et al. ${ }^{22}$. A prática regular de exercícios físicos promove aumento da $\mathrm{RC}$, diminuindo a FC de repouso e aumentando a FC de pico ${ }^{11,12}$. Além disso, nossos resultados mostram aumento no pulso de oxigênio na intensidade do LA após RCV, indicando melhora da eficiência do sistema cardiovascular, pois expressa o consumo de $\mathrm{O}_{2}$ pelo organismo a cada batimento cardíaco ${ }^{13,21}$.

Outro resultado importante do presente foi o ganho médio de 21 $\mathrm{N} / \mathrm{m}$ no pico de torque dos músculos extensores de joelho. Gary et al. ${ }^{23}$ desenvolveram um programa de exercícios aeróbicos e resistidos durante 12 semanas em pacientes com IC e relataram aumento na distância percorrida no teste de caminhada de 6 minutos e da força dos músculos extensores de joelho. A ênfase no treino de músculos dos membros inferiores pode contribuir com a melhora na capacidade de exercício e na qualidade de vida de pacientes com $\mathrm{IC}^{24}$. Além disso, a participação efetiva de pacientes em programas de reabilitação cardiovascular pode promover desfechos clínicos favoráveis, incluindo melhora da função vascular e qualidade de vida, reduzindo os riscos de hospitalização ou morte ${ }^{10,25}$. Beckers et al. ${ }^{26}$ realizaram estudo por um período de 6 meses com protocolo de exercício aeróbico e resistido e observaram melhora da capacidade submáxima de esforço, da força da musculatura periférica e redução dos sintomas relacionados à patologia, corroborando com os nossos achados.

O questionário WHOQOL-bref vem sendo utilizado para avaliar os efeitos de protocolos de exercícios em pacientes IC ${ }^{27}$. Nossos resultados corroboram com os achados de Bocalini et al. ${ }^{27}$ que mostraram melhora no domínio psicológico do WHOQOL-bref. Este domínio está relacionado com sentimentos, aprendizagem, memória e atenção, espiritualidade e pensamentos positivos ou negativos ${ }^{16}$. Acreditamos que o aumento significativo neste domínio está relacionado à socialização e convívio com diferentes pessoas durante o protocolo de treinamento. Além disso, os programas de RCV podem atuar como ferramentas úteis na quebra da rotina desses pacientes, promovendo mudanças em suas atividades de vida diária, gerando hábitos saudáveis e diminuindo o risco de eventos cardiovasculares ${ }^{10,25}$

Em nosso estudo, o aumento da VE pós RCV foi observado no LA e no esforço máximo. Entretanto, não observou-se diferenças pré e pós $\mathrm{RCV}$ na relação $\mathrm{VE} / \mathrm{VCO}_{2}$, um indicador de eficiência ventilatória. Winkelmann et al. ${ }^{28}$, relataram aumento na VE após um programa que associou exercícios aeróbicos com o treinamento muscular inspiratório em pacientes com IC. Acredita-se que o treinamento físico possa promover melhora da função diafragmática e redução da congestão pulmonar ${ }^{29}$. Em outro estudo, os pesquisadores relataram redução na VE durante protocolo com carga constante após treinamento físico em pacientes $\mathrm{IC}^{20}$.

\section{CONCLUSÃO}

Um programa de 24 sessões de RCV promove melhora da capacidade funcional submáxima, aumenta o pico de torque dos extensores de joelho e melhora o domínio psicológico do questionário de qualidade de vida em pacientes com IC. A inserção de pacientes portadores de IC em programas de RCV deve ser considerada um complemento à terapia farmacológica pelo cardiologista.

Todos os autores declararam não haver qualquer potencial conflito de interesses referente a este artigo.

CONTRIBUIÇÕES DOS AUTORES: Cada autor contribuiu individual e significativamente para o desenvolvimento do manuscrito. LC (0000-0003-0758-3467)* e JBCR (00000002-3382-1919)* responsáveis pelo planejamento dos métodos, análise estatística e escrita do manuscrito. BFB (0000-0001-5080-7089)*, JB (0000-0002-8198-4202)*, SR (0000-0001-9502-2736)*, GFF (0000-0003-3026-5950)* e MC (0000-0002-6942-4034)* responsáveis pela execução do projeto, coleta dos dados e escrita do manuscrito. GLP (0000-0002-2263-3189)* realizou a revisão crítica do manuscrito e contribuiu com o conceito intelectual do estudo. *ORCID (Open Researcher and Contributor ID).

\section{REFERÊNCIAS}

1. Go AS, Mozaffarian D, Roger VL, Benjamin EJ, Berry JD, Blaha MJ, et al. Executive summary: heart disease and stroke statistics - 2014 update: a report from the American Heart Association. Circulation. 2014;129(3):399-410.
2. Bocchi EA, Marcondes-Braga FG, Bacal F, Ferraz AS, Albuquerque D, Rodrigues DA, et al. Updating of the Brazilian guideline for chronic heart failure - 2012. Arq Bras Cardiol. 2012;98(1 Suppl 1):1-33.

3. Gravina CF, Rosa RF, Franken RA, Freitas EV, Liberman A, Nussbacher A, et al. Sociedade Brasileira de 
Cardiologia. II Diretrizes Brasileiras em Cardiogeriatria. Arq Bras Cardiol 2010; 95(3 Supl. 2):1-112

4. Arena R, Myers J, Guazzi M. The clinical and research applications of aerobic capacity and ventilatory efficiency in heart failure: an evidence-based review. Heart Fail Rev. 2008;13(2):245-69.

5. Magnusson G, Isberg B, Karlberg KE, Sylvén C. Skeletal muscle strength and endurance in chronic congestive heart failure secondary to idiopathic dilated cardiomyopathy. Am J Cardiol. 1994;73(4):307-9.

6. Wilson JR, Mancini DM. Factors contributing to the exercise limitation of heart failure. J Am Coll Cardiol. 1993;22(4 Suppl A):93A-8.

7. Aikawa P, Cintra ARS, Oliveira Júnior AS, Silva CTM, Pierucci JD, Afonso MS, et al. Reabilitação cardíaca em pacientes submetidos a cirurgia de revascularização do miocárdio. Rev Bras Med Esporte. 2014;20(1):55-8

8. Leon AS, Franklin BA, Costa F, Balady GJ, Berra KA, Stewart KJ, Cardiac rehabilitation and secondary prevention of coronary heart disease: an American Heart Association scientific statement from the Council on Clinical Cardiology (Subcommittee on Exercise, Cardiac Rehabilitation, and Prevention) and the Council on Nutrition, Physical Activity, and Metabolism (Subcommittee on Physical Activity), in collaboration with the American association of Cardiovascular and Pulmonary Rehabilitation. Circulation. 2005;111(3):369-76.

9. Ades PA, Green NM, Coello CE. Effects of exercise and cardiac rehabilitation on cardiovascular outcomes. Cardiol Clin. 2003;21(3):435-48.

10. Herdy AH, López-Jimenez F, Terzic CP, Milani M, Stein R, Carvalho T; Sociedade Brasileira de Cardiologia. Diretriz Sul-Americana de Prevenção e Reabilitação Cardiovascular. Arq Bras Cardiol 2014;103(2 Supl.1):1-31.

11. Bennett JA, Riegel B, BittnerV, Nichols J.Validity and reliability of the NYHA classes for measuring research outcomes in patients with cardiac disease. Heart Lung. 2002;31(4):262-70.

12. Arena R, Myers J, Guazzi M. The clinical importance of cardiopulmonary exercise testing and aerobic training in patients with heart failure. Rev Bras Fisioter. 2008;12(2):75-87.

13. Neder JA, Nery, LE. Fisiologia clínica do exercício: teoria e prática. 1a. ed. São Paulo: Artes Médicas; 2003.

14. Meneghelo RS, Araújo CGS, Stein R, Mastrocolla LE, Albuquerque PF, Serra SM, et al. Sociedade Brasileira de Cardiologia. III Diretriz da Sociedade Brasileira de Cardiologia sobre Teste Ergométrico. Arq Bras Cardiol. 2010; 95(5):1-26.

15. Degache F, Garet M, Calmels P, Costes F, Bathélémy JC, Roche F. Enhancement of isokinetic muscle strength with a combined training programme in chronic heart failure. Clin Physiol Funct Imaging. 2007;27(4):225-30

16. Fleck MP, Louzada S, Xavier M, Chachamovich E, Vieira G, Santos L, Pinzon V. Application of the Portuguese version of the abbreviated instrument of quality life WHOQOL-bref. Rev Saude Publica. 2000;34(2):178-83.

17. Selig SE, Levinger I, Williams AD, Smart N, Holland DJ, Maiorana A, et al. Exercise \& Sports Science Australia Position Statement on exercise training and chronic heart failure. J Sci Med Sport. 2010;13(3):288-94.

18. Pereira DAG, Rodrigues RS, Samora GAR, Lage SM, Alencar MCN, Parreira VF, et al. Capacidade funcional de indivíduos com insuficiência cardíaca avaliada pelo teste de esforço cardiopulmonar e classificação da New York Heart Association. Fisioter Pesq. 2012;19(1):52-6.

19. Weber KT, Kinasewitz GT, Janicki JS, Fishman AP. Oxygen utilization and ventilation during exercise in patients with chronic cardiac failure. Circulation. 1982;65(6):1213-23.

20. Sullivan MJ, Higginbotham MB, Cobb FR. Exercise training in patients with chronic heart failure delays ventilatory anaerobic threshold and improves submaximal exercise performance. Circulation. 1989;79(2):324-9.

21. Negrão CE, Barreto AC. Cardiologia do exercício: do atleta ao cardiopata. 3a. ed. Barueri, SP: Manole; 2010.

22. Colucci WS, Ribeiro JP, Rocco MB, Quigg RJ, Creager MA, Marsh JD, et al. Impaired chronotropic response to exercise in patients with congestive heart failure. Role of postsynaptic beta-adrenergic desensitization. Circulation. 1989;80(2):314-23

23. Gary RA, Cress ME, Higgins MK, Smith AL, Dunbar SB. Combined aerobic and resistance exercise program improves task performance in patients with heart failure. Arch Phys Med Rehabil. 2011;92(9):1371-81.

24. Hambrecht R, Gielen S, Linke A, Fiehn E, Yu J, Walther C, et al. Effects of exercise training on left ventricular function and peripheral resistance in patients with chronic heart failure: a randomized trial. JAMA. 2000;283(23):3095-101.

25. O'Connor CM, Whellan DJ, Lee KL, Keteyian SJ, Cooper LS, Ellis SJ, et al. Efficacy and safety of exercise training in patients with chronic heart failure: HF-ACTION randomized controlled trial. JAMA. 2009;301(14):1439-50.

26. Beckers PJ, Denollet J, Possemiers NM, Wuyts FL, Vrints CJ, Conraads VM. Combined endurance-resistance training vs. endurance training in patients with chronic heart failure: a prospective randomized study. Eur Heart J. 2008;29(15):1858-66.

27. Bocalini DS, dos Santos L, Serra AJ. Physical exercise improves the functional capacity and quality of life in patients with heart failure. Clinics (Sao Paulo). 2008;63(4):437-42.

28. Winkelmann ER, Chiappa GR, Lima CO, Viecili PR, Stain R, Rbeiro JP. Addition of inspiratory muscle training to aerobic training improves cardiorespiratory responses to exercise in patients with heart failure and inspiratory muscle weakness. Am Heart J. 2009;158(5):768.e1-7.

29. Zurek M, Corra U, Piepoli MF, Binder RK, Saner H, Schmid JP. Exercise training reverses exertional oscillatory ventilation in heart failure patients. Eur Respir J. 2012;40(5):1238-44. 